\title{
Osmotic Activity
}

National Cancer Institute

\section{Source}

National Cancer Institute. Osmotic Activity. NCI Thesaurus. Code C40526.

Osmotic Activity is a passive process in which water diffuses across a cell membrane in response to a concentration gradient. 